\title{
A NOTE ON THE MINIMUM OF A BINARY FORM
}

\author{
N. C. ANKENY
}

Let $f(x, y)=a_{0} x^{n}+a_{1} x^{n-1} y+\cdots+a_{n} y^{n}$ be a binary form of degree $n$ with real coefficients. The simplest algebraic invariant of such a form is its discriminant, defined by

$$
D(f)=a_{0}^{2(n-1)} \prod_{i<j}\left(\theta_{i}-\theta_{j}\right)^{2},
$$

where ${ }^{1}$

$$
f(x, y)=a_{0}\left(x-\theta_{1} y\right) \cdots\left(x-\theta_{n} y\right) .
$$

$D(f)$ is a homogeneous polynomial of degree $2(n-1)$ in $a_{0}, \cdots, a_{n}$. The simplest arithmetical invariant of such a form is its minimum $L(f)$, defined as the lower bound of $|f(x, y)|$ for all integral values of $x, y$ except 0,0 .

For $n=2$ and 3 , it is known that if $D(f) \neq 0$, then

$$
L^{2(n-1)}(f) \leqq c|D(f)|,
$$

where $c$ is a constant. The best possible value of $c$ is known for each type of form with $n=2$ or 3 . We say that a form is of type $(r, s)$ if $\theta_{1}, \cdots, \theta_{n}$ consist of $r$ real numbers, and $s$ pairs of conjugate complex numbers (so that $r+2 s=n$ ). If we denote by $c(r, s)$ the least value of $c$ for which (3) holds, then

$$
c(0,1)=1 / 3, \quad c(2,0)=1 / 5, \quad c(3,0)=1 / 49, \quad c(1,1)=1 / 23 .
$$

The first two results are classical, and the last two are due to Mordell.

The object of this note is to prove that no inequality of the type (3) is valid for all binary forms of degree $n$, and of a given type, when $n$ is greater than 3. This fact is probably well known to those who have followed recent work on the geometry of numbers, but no simple direct proof seems to have been given. One argument which suggests why (3) cannot be expected to hold when $n>3$ is the following. When $n=2$ or 3 , a corollary to the existing results tells us that if $D(f)=0$ then $L(f)=0$, that is, $f(x, y)$ then takes values which are arbitrarily small numerically. This is not true for $n=4$ as we see from the simple example $\left(x^{2}-2 y^{2}\right)^{2}$. We shall show that if $n>3$ there exist

Received by the editors May 13, 1948.

1 The definition fails if $a_{0}=0$. In this case, we must first transform $f$ by a linear substitution of determinant \pm 1 into a form whose leading coefficient is not zero. 
forms for which $|D(f)|$ is arbitrarily small, but not zero, and $L(f)$ is not small.

I am indebted to Prof. Harold Davenport for suggesting this topic to me and to both him and Prof. L. J. Mordell for comments on the manuscript.

In what follows, all small Latin letters denote rational integers. We recall that any irrational number $\alpha$, with $1<\alpha<2$, has a continued fraction representation

$$
\alpha=1+\frac{1}{a_{1}}+\frac{1}{a_{2}}+\cdots
$$

where $a_{1}, a_{2}, \cdots$ are positive. If $p_{n} / q_{n}$ is the $n$th convergent to $\alpha$, so that

$$
\frac{p_{n}}{q_{n}}=1+\frac{1}{a_{1}}+\cdots+\frac{1}{a_{n}},
$$

we have the following well known results:

LEMMA 1.

$$
\frac{1}{3 a_{n+1} q_{n}^{2}}<\left|\alpha-\frac{p_{n}}{q_{n}}\right|<\frac{1}{a_{n+1} q_{n}^{2}} .
$$

LEMmA 2. If $q_{n} \leqq y<q_{n+1}$, then $\left|p_{n}-\alpha q_{n}\right| \leqq|x-\alpha y|$ for all $x$.

Lemmas 1 and 2 imply:

LeMmA 3. If $q_{n} \leqq y<q_{n+1}$, then $|x-\alpha y|>1 / 3 a_{n+1} q_{n} \geqq 1 / 3 a_{n+1} y$ for all $x$.

We now prove:

LemMA 4. If $\alpha>1$ and $\beta>1$ are both irrationals whose continued fraction expansions have all their partial quotients less than $N$, then

$$
|(x-\alpha y)(x+\beta y)|>1 / 3 N
$$

for all $x, y$ not both 0 .

Proof. We may certainly assume $x y \neq 0$. Suppose first $x y>0$. Then $|x+\beta y|>|\beta y|>|y|$. Also, $|x-\alpha y|>1 / 3 N|y|$ from Lemma 3, so the result follows by multiplying these two inequalities. The case when $x y<0$ follows from the case $x y>0$ on interchanging $\alpha$ and $\beta$.

Lemma 5. Let $\theta$ be a real quadratic irrational integer, and let $\theta^{\prime}$ be its conjugate. Suppose $\theta>1$ and $\theta^{\prime}>1$. Let $\alpha$ satisfy the condition of Lemma 


\section{Then}

$$
\left|(x-\alpha y)(x+\theta y)\left(x+\theta^{\prime} y\right)\right|>1 / 3 N
$$

for all $x, y$ not both zero.

Proof. Again we may assume $x y \neq 0$. If $x y<0$, we have $|x-\alpha y|>1$, and $\left|(x+\theta y)\left(x+\theta^{\prime} y\right)\right| \geqq 1$ since it is the absolute norm of an algebraic integer not zero. If $x y>0,|x+\theta y|>|y|,\left|x+\theta^{\prime} y\right|>|y|$ and $|x-\alpha y|$ $>1 / 3 N|y|$ by Lemma 3 . This proves the result.

We can now prove the main theorem by constructing forms $F(x, y)$ of given type $(r, s)$ where $r+2 s=n \geqq 4$.

Case $1 . r \geqq 4, r \neq 5$. Let $G(x, y)$ be any binary form of type $(r-4, s)$ with integral coefficients, and $D(G) \neq 0$, such that the $r-4$ real roots of $G(\xi, 1)=0$ are all irrational. Since $r-4 \neq 1$; such a form can be constructed by multiplying together various forms of the type

$$
x^{2}-m y^{2}, \quad x^{2}+m y^{2}, \quad x^{3}+2 x^{2} y-x y^{2}-y^{3}
$$

using several different values of $m$ if necessary, and not more than one cubic form. (If $r=4, s=0$, we take the empty product, that is, $G(x, y)=1$.) Then $L(G) \geqq 1$. Let

$$
F(x, y)=\left(x-\alpha_{1} y\right)\left(x+\beta_{1} y\right)\left(x-\alpha_{2} y\right)\left(x+\beta_{2} y\right) G(x, y)
$$

where $\alpha_{1}, \beta_{1}$ and $\alpha_{2}, \beta_{2}$ satisfy the hypotheses of Lemma 4 and $\alpha_{1} \neq \alpha_{2}$, $\beta_{1} \neq \beta_{2}$, and none of $\alpha_{1}, \alpha_{2},-\beta_{1},-\beta_{2}$, coincides with a root of $G(\xi, 1)=0$, Then

$$
L(F) \geqq(3 N)^{-2}
$$

by Lemma 4. But, if the $\alpha$ 's and $\beta$ 's are bounded,

$$
0<|D(F)|<A(G)\left(\alpha_{1}-\alpha_{2}\right)^{2}\left(\beta_{1}-\beta_{2}\right)^{2}
$$

where $A(G)$ is bounded for fixed $G$ if the $\alpha$ 's and $\beta$ 's are bounded.

We fix $\alpha_{1}, \beta_{1}, \beta_{2}$ and choose $\alpha_{2}$ to have the same first $k+1$ partial quotients as $\alpha_{1}$. Then by Lemma 1 ,

$$
\left|\alpha_{1}-\alpha_{2}\right| \leqq\left|\alpha_{1}-\frac{p_{k}}{q_{k}}\right|+\left|\alpha_{2}-\frac{p_{k}}{q_{k}}\right|<\frac{2}{a_{k+1} q_{k}^{2}} \leqq \frac{2}{k^{2}} .
$$

Hence $D(F) \rightarrow 0$ as $k \rightarrow \infty$, which proves the result.

Case 2. $r=5$. Let $G(x, y)$ be a form as above, of type $(0, s)$, which can be taken to be a product of forms $x^{2}+m y^{2}$. Then $L(G) \geqq 1$. Let

$$
F(x, y)=\left(x-\alpha_{1} y\right)(x+\theta y)\left(x+\theta^{\prime} y\right)\left(x-\alpha_{2} y\right)\left(x+\beta_{2} y\right) G(x, y),
$$

where $\alpha_{1}, \alpha_{2}, \beta_{2}$ satisfy the same hypotheses as before and $\theta$ satisfies 
the hypotheses of Lemma 5 , and $-\theta,-\theta^{\prime}, \alpha_{1}, \alpha_{2},-\beta_{2}$ are distinct. By Lemmas 4 and 5 ,

$$
L(F) \geqq(3 N)^{-2} .
$$

Again $D(F)$ may be made arbitrarily small, but not zero, by choice of $\alpha_{2}$.

Case 3. $r \leqq 3$. This presents less difficulty, and we take $F(x, y)$ to have the following form:

$$
\begin{aligned}
r=0, s \geqq 2, \quad F(x, y) & =\left(x^{2}+y^{2}\right)\left(x^{2}+(1+\epsilon) y^{2}\right) G(x, y), \\
L(F) & \geqq L(G) \geqq 1, \\
r=1, s \geqq 2, \quad F(x, y) & =(x-\alpha y)\left(x^{2}+y^{2}\right)\left(x^{2}+(1+\epsilon) y^{2}\right) G(x, y), \\
|F(x, y)| & \geqq \frac{1}{3 N|y|} y^{2} \geqq \frac{1}{3 N} \quad \text { for } y \neq 0, \\
r=2, s \geqq 1, \quad F(x, y) & =\left(x-\alpha_{1} y\right)\left(x-\alpha_{2} y\right)\left(x^{2}+y^{2}\right) G(x, y), \\
|F(x, y)| & \geqq \frac{1}{(3 N y)^{2}} y^{2}=\frac{1}{(3 N)^{2}} \quad \text { for } y \neq 0, \\
r=3, s \geqq 1, \quad F(x, y)=\left(x-\alpha_{1} y\right)\left(x+\beta_{1} y\right)\left(x-\alpha_{2} y\right)\left(x^{2}+y^{2}\right) G(x, y), & \text { for } y \neq 0 .
\end{aligned}
$$

In the first two cases $D(F) \rightarrow 0$ with $\epsilon$; in the other two cases $D(F)$ is arbitrarily small, but unequal to zero, by choice of $\alpha_{2}$.

STANFORD UNIVERSITY 